Inge Voller*

\title{
Die Modalpartikeln auf Deutsch und Dänisch - eine kontrastive Analyse
}

\begin{abstract}
This article is about the German and Danish modal particles in a contrastive analysis. There are several features, which are characteristic for this particular word class. After a few comments on those the article is concerned about how to make a proper analysis of those words based on the German modal particles. As shall be shown these words are important to a communication situation, because they show the interlocutors how they are supposed to understand each other. This analysis will be an approach finding out, which functions these words have and the point is, that it is possible to find appropriate equivalents in Danish for the German modal particles, which can be listed, maybe in a dictionary.
\end{abstract}

\section{Einleitung}

Aus mehreren Gründen haben die Modalpartikeln (nachfolgend nur: MP) erst richtig in den letzten Jahrzehnten die Forschung interessiert, und viele neue Ergebnisse sind in dieser Zeitspanne aufgetaucht - besonders über die deutschen MP. Und da diese Elemente in der Sprache erstens eine wichtige Rolle für die Kommunikation spielen und zweitens für Deutsch lernende Ausländer ein schwieriges Gebiet ausmachen, wäre es sinnvoll mit einer Wortliste über die deutschen und dänischen Entsprechungen innerhalb dieser Wortklasse. Deshalb folgt hier eine kurze Analyse, wie man sich mit den MP in einer kontrastiven Analyse auseinander setzen und dadurch eine Wortliste aufstellen kann, die den dänischen Schülern/ Studierenden die Arbeit mit den MP erleichtern sollte. Eine solche Wortliste ist mir bekannt nie früher gemacht worden, u.a. deshalb weil die dänischen MP nicht in einem so hohen Grad erforscht worden sind wie die deutschen. Das Ziel sollte also sein, alle deutschen MP so

\footnotetext{
* Inge Voller

Rolfsvej 3. th.

DK-3000 Helsingør
}

Hermes, Journal of Linguistics no. 28-2002 
136

zu untersuchen, dass jede deutsche MP eine Entsprechung auf Dänisch hat. Diese Entsprechung kann dann entweder eine dänische MP, ein ganz anderer Ausdruck, eine Paraphrase usw. sein.

\section{Besondere Kennzeichen der deutschen MP}

In der Analyse muss man erst feststellen, was eine MP ist, um die Auswahl der deutschen MP richtig zu treffen. Da die MP kommunikationslenkende Elemente sind, gelten bestimmte Kriterien für die Untersuchung der grammatischen Eigenschaften. Erstens muss betont werden, dass man sie von dem Kontext nie losgerissen und jeder für sich alleine betrachten kann. Alle MP haben nämlich Homonyme in anderen Wortklassen, was die Untersuchung erschwert. Demzufolge muss man sich immer den Kontext/ die Kommunikationssituation vor Auge halten, wenn man sie erforschen soll. Zweitens kann man in der konkreten Analyse festzustellen versuchen, was den Unterschied zwischen einem Satz mit MP und ohne MP ausmacht bzw. zwischen demselben Satz mit einer MP und mit einer anderen MP. Das wären die so genannten Deletions- und Substitutionstests (Helbig 1977: 35).

Außerdem können die MP unterschiedliche Funktionen haben, je nachdem in welchen Satztypen sie vorkommen. Das heißt, dass man zwischen lokutiven und illokutiven Verhältnissen unterscheidet. Derselbe lokutive Satz kann mehrere verschiedene illokutive Varianten haben (Helbig 1994: 58). Die illokutiven Varianten unterscheiden sich auch oft aufgrund der unterschiedlichen Satztypen, warum man sie in der Analyse mit in Betracht ziehen sollte.

\subsection{Kommunikative-pragmatische Merkmale}

Man könnte die MP auf allen Ebenen der Sprache untersuchen, und sie haben natürlich auch besondere Merkmale auf allen Ebenen, aber die größte Rolle spielen sie auf der kommunikativ-pragmatischen Ebene. Sie dienen der Färbung des Satzes dadurch, dass sie einen persönlich, subjektiv geprägten Kontakt zum Gesprächspartner herstellen. Ohne MP ist ein Satz eher neutral, während ein Satz mit MP gefärbt ist:

(1) Wie heißt du?

(2) Wie heißt du denn? 
Der Letzte Satz drückt eher Teilnahme aus als der erste. Das persönliche Interesse des Sprechers scheint durch dieses kleine Wort denn durch. Das Interesse kann sich u.a. dadurch zeigen, dass der Sprecher an ein gemeinsames Wissen anspielt, wie im folgenden Satz:

(3) Das weißt du doch!

Mit dieser Aussage wird angedeutet, dass der Empfänger schon das Gesagte weiß: Er muss nur daran erinnert werden. Das Gleiche wäre mit einer Aussage ohne MP nicht der Fall gewesen, wenn man es mit anderen Mitteln (z.B. Betonung, Mimik usw.) nicht deutlich macht (Kirstein 1983: 217).

Die bekannteste Definition der MP-Funktion stammt von Weydt (1969: 60): Die MP erzählen dem Empfänger etwas über das Verhalten des Sprechers zu seiner Aussage, und auch wie der Empfänger sich zur Aussage verhalten soll. Sie machen darauf aufmerksam, dass man sich überhaupt in einer Kommunikationssituation befindet, da sie Signale zum Empfänger sind. Weil sie das Verhalten des Sprechers zu seiner Aussage ausdrücken, sind sie sehr nützlich in einer Kommunikationssituation: Sie ersparen dem Sprecher viele Worte, die gegebenenfalls dem Empfänger zur Kenntnis geben sollten, wie er die Aussage aufzufassen hat (Krivonosov 1983: 40-45).

Das könnte am folgenden Beispiel illustriert werden:

(4) Sie ist doch schön.

In einem Satz mit doch, wird dem Empfänger erzählt, dass er beizustimmen hat.

Wenn der Empfänger eine solche Aussage verneinen würde, müsste er ausführlichere Auskunft geben, warum er dieser Meinung ist. Die MP kodiert den Empfänger, damit er keine kommunikativen „Fehler" macht. Es wird erwartet, dass er auf Satz (4) zustimmend reagiert.

So könnten auch viele andere kommunikativ-pragmatische Merkmale beschrieben werden, aber da das nicht das Hauptziel dieses Aufsatzes ist, belasse ich es hier bei diesen einleitenden Betrachtungen.

\section{Statistische Ergebnisse}

Um darüber im Klaren zu sein, wie viele dänische MP es im Vergleich zu deutschen MP gibt, habe ich eine Aufzählung deutscher bzw. dänischer 
MP in Zeitungen vorgenommen. Alle deutschen MP wurden in „Die Zeit" vom 28.10.99 gefunden, und alle dänischen MP wurden in „Politiken" vom bzw. 15.11.99 und 16.11.99 gefunden. Die ganzen Zeitungen sind durchgenommen worden außer Werbungen, Anzeigen und Sportabteilen. Es ist in der Sprachwissenschaft zwar betont worden, dass die MP eher in der gesprochenen Sprache gebraucht werden, weil man sich da unmittelbarer und unüberlegter zu seinem Gesprächspartner hinwendet (Kriwonossow 1977: 4). Wie oben erwähnt, hängt die Verwendung von MP mit dem Grad der Intimität der Interaktionspartner zusammen, und da man sich schriftlich (sprich: in Zeitungen) eher formell mit sachlichen Auskünften an den Leser wendet, braucht man demzufolge weniger MP, als wenn man Gesicht zu Gesicht steht.

Andersherum sind die MP in geschriebener Sprache die einzigen Faktoren, die die Aspekte wahrnehmen, die in gesprochener Sprache durch Intonation, Mimik usw. gezeigt werden.

Diese Aufzählung wurde deswegen vorgenommen, weil MP-Forscher (u.a. Weydt 1969: 11) darauf aufmerksam gemacht haben, dass das Deutsche mehr MP hat als andere Sprachen, so z. B. Französisch und Englisch. Also wäre es interessant herauszufinden, wie es sich auf Dänisch verhält.

Ich habe die Artikel der Zeitungen in zwei Bereiche aufgeteilt: Artikel, die als Schriftsprache bezeichnet werden können (Berichte), und Artikel, die der wiedergegebenen gesprochenen Sprache angehören (Interviews, direkte Rede). In den jeweiligen Kategorien habe ich 1000 deutsche Wörter und 1000 dänische Wörter aus verschiedenen Artikeln aufgezählt, um auszuschließen, dass das Vorkommen der MP in Verbindung mit der Schreibweise des jeweiligen Autors steht.

In den geschriebenen Artikeln stellt es sich heraus, dass es pro 1000 deutsche Wörter 15 MP gibt, während das Dänische 14 MP aufweist. Wenn das repräsentativ für die Verteilung der MP in der deutschen und dänischen Sprache ist, ist der Unterschied nicht groß.

In den der wiedergegebenen gesprochenen Sprache angehörigen Artikeln gibt es auf Dänisch $30 \mathrm{MP}$ pro 1000 Wörter, während man auf Deutsch „nur“ 25 vorfindet. Das ist ein nicht-erwartetes Ergebnis, wenn das repräsentativ ist. Gründe können sein, dass man in der deutschen Umgangssprache mehr MP braucht, und dass die wiedergegebene Sprache in der Zeitung nicht so wiedergegeben ist, wie die Deutschen 
sprechen, wogegen die dänische gesprochene Sprache besser wiedergegeben ist. Ein anderer Grund kann sein, dass die dänische Umgangssprache immer mehr MP als das Deutsche verwendet hat, aber keiner hat es aufgezählt.

Ich habe auch aufgezählt, wie viele verschiedene MP es in den beiden Sprachen gibt: Auf Deutsch gibt es 40 MP. Im Dänischen gibt es in den Zeitungen 38 solche Wörter. Auch hier gibt es also keinen beeindruckenden Unterschied. Doch gibt es einen Unterschied in Bezug auf die Frequenz: Auf Dänisch wird jo ca. 23 \% der Male eingesetzt, während die anderen MP zwischen 1-9 \% der Male verwendet werden. Auf Deutsch sieht es so aus, als ob man ein breiteres Spektrum der MP braucht: Alle MP werden zwischen 1-12\% der Male verwendet. Allerdings gibt es in beiden Sprachen eine große Gruppe, die fast nie gebraucht wird (sie werden um $1 \%$ der Male herum gebraucht).

\section{Die Analyse}

Im Folgenden werden ein paar ausgewählte deutsche MP analysiert. In der deutschen Zeitung (siehe oben) habe ich alle MP beinhaltenden Sätze gefunden und Beispielsätze ausgewählt. Anhand derer werde ich versuchen, die Funktion der MP herauszufinden, und ausgehend davon finde ich dann mögliche Entsprechungen im Dänischen. Die Funktion der deutschen MP wird nicht immer durch eine dänische MP wahrgenommen, sondern vielleicht durch eine Frage, ein Modalwort, Negationswörter usw. (Andersen 1989: 21). Die Fragestellung dieser Analyse lautet: Wie drückt man diesen Sachverhalt in einer analogen Situation auf Dänisch aus? (Heinrichs 1981: 1903).

Außerdem werden die Analysen je nach Satztyp aufgeteilt, da die MP, wie oben erwähnt, mit verschiedenen Satztypen ihre Funktion und ihren Inhalt ändern können. Die Beispiele sind vorne in den Kapiteln aufgeführt, und im Text wird nur zu den Beispielnummern hingewiesen. Wenn eine Seitenzahl hinter dem Beispiel steht, verweist auf die gegebene Seite in „Die Zeit“. Wenn nichts dahinter steht, sind die Beispiele frei erfunden. Die Beispiele mit hinten angestellten kleinen Buchstaben sollen Äquivalenzen zum ursprünglichen Beispiel darstellen. Das Beispiel (5a) soll somit die zu (5) passende Übersetzung sein. 


\subsection{Denn}

(5) Wann können Ihre Kunden denn mit weiteren Tarifsenkungen der Telekom rechnen? (S. 25)

(6) Was gibt's denn hier im Wald für die Jugend? (S. 19).

(7) Wer kümmert sich denn darum?

(8) Was ist denn mit dem los? (S. 16)

(9) Sind denn da Fische drin? (S. 19)

(10) Bist du denn verrückt geworden?

Die MP denn ist in der Sprachwissenschaft längst zur ,Interrogativpartikel“ erwählt worden. Sie kommt fast ausschließlich in Interrogativsätzen vor.

\subsubsection{Ergänzungsfragen}

Wenn denn unbetont in einer Ergänzungsfrage verwendet wird, ist die Funktion der MP den Empfänger dem Sprecher gegenüber freundlicher zu stimmen. Eine Frage mit denn scheint viel freundlicher und persönlicher als eine Frage ohne MP. Dasselbe gilt, wenn man mit der Frage seine Kritik oder Sorge ausdrücken möchte: Die Frage scheint weniger hart mit MP als ohne. Gleichzeitig ist denn Ausdruck dafür, dass die Frage sich auf die vorhandene Thematik und Situation bezieht, und damit legt die MP die Frage im Kontext fest (Becker 1976: 8). Diese offenkundige Tatsache ist u.a. durch (5) zu erkennen, da diese Frage in allen anderen Zusammenhängen als in einer Diskussion über die Firmenpolitik der Telekom sinnlos gewesen wäre.

Dittmann (1980: 63-64) zufolge hat denn die Fähigkeit, eine normale Frage zu einer Kritik zu drehen, macht es aber nicht immer. Das hängt vom Kontext ab. Natürlich hängt die Funktion der MP immer von dem Kontext und dem semantischen Inhalt des Textes ab, aber wenn eine MP nur dann ein Zeichen der Kritik ist, wenn diese in einem kritischen Satz eingebettet ist, dann hat man zu viel in die Funktion der MP hineingelegt. Die Kritik/Besorgnis hat dann nichts mehr mit der MP zu tun, sondern mit der Aussage des Satzes. Bei einem Satz wie (6) sollte man demzufolge auch den eventuellen kritischen Ton des Satzes nicht der MP sondern vielmehr dem Sachverhalt zuschreiben.

Wenn denn verwendet wird, wird die Frage gestellt, damit man sich in das gerade besprochene Thema vertiefen soll (König 1977: 121). Im 
Gegensatz zu eigentlich knüpft also denn zu dem aktuellen Thema an, wo ein Satz mit eigentlich eher ein neues Thema anschlagen soll. Die Situation zu der ein solcher Satz mit denn anknüpfen soll, muss nicht notwendigerweise eine konkret ausgesprochene Aussage sein, es kann sich auch um eine nicht-sprachliche Handlung drehen. Eine Frage mit denn bezieht sich also immer auf die aktuelle Situation, aber die Frage wird nicht immer gestellt, damit man eine Antwort bekommen soll. Die Frage kann auch rein rhetorischer Art sein, wie im Beispiel (7). Der Sprecher nimmt an, dass seine Auffassung allgemein geteilt wird.

Diese Funktionen werden auf Dänisch auch durch MP wahrgenommen, und zwar durch entweder så oder $d a$. Letztgenannte ist am meisten brauchbar in Fragen rhetorischer Art, während beide in normalen Ergänzungsfragen verwendet werden können:

(5a) Hvornår kan Deres kunder så regne med yderligere tarifnedsættelser fra Telekoms side?

Man könnte auch dog in der nicht-betonten Version gebrauchen, und eine ganz andere Möglichkeit wäre, den ganzen Satz emphatisch umzuschreiben, damit die Hervorhebung der Frage durch denn im Dänischen die Form eines Nebensatzes hat:

(6a) Hvad er det, der er her i skoven for de unge?

Die rhetorische Variante könnte auch zu einer durativen Form umgeschrieben werden. Die durative Form kommt auf Dänisch durch zwei Verben zustande, wovon das eine nur die Funktion der Duration übernimmt, während das andere die tatsächliche Handlung beschreibt. Die Durationsform wird auf Englisch durch eine -ingform übernommen:

(7a) Who is worrying about that?

(7b) Hvem går (da) rundt og bekymrer sig om det?

Wenn denn betont ist, gelten dieselben Kriterien wie für das unbetonte denn: Es drückt Überraschung und Freundlichkeit aus, legt den Satz im Kontext fest, ist ein illokutiver Faktor der Frage, aber das betonte denn kommt nur da vor, wo der Sprecher seine bisherigen Informationen verifizieren bzw. berichtigen möchte. Denn drückt aus, dass der Empfänger die Informationen verstanden hat, aber dass sie nicht genügend waren (Dittmann 1980: 68 og Abraham 1986: 6). Denn ist ein Zeichen, dass der Empfänger nicht die erwarteten Informationen bekommen hat. (8) könnte betont werden: 
142

a. A denkt, dass $\mathrm{C}$ müde ist, weil er die ganze Nacht Computer gespielt hat.

b. B sagt, dass $\mathrm{C}$ nicht deswegen müde ist.

c. A stellt die Frage (8).

d. B beantwortet, dass $\mathrm{C}$ krank geworden und deswegen müde ist.

Auf Dänisch würde man hier ein (eventuell betontes) så brauchen. Es hebt hervor, dass schon ein Erklärungsversuch unternommen worden ist, und mit der så beinhaltenden Frage wird ein anderer Grund erfragt.

\subsubsection{Entscheidungsfragen}

Denn in einer Entscheidungsfrage knüpft auch an das Vorangegangene an, egal ob es von verbaler oder nicht-verbaler Art ist. Die MP stellt die Frage in einem kausal-logischen Zusammenhang mit der den beiden Gesprächspartnern bekannten Kommunikationssituation, so dass beide Gesprächspartner wissen, in welchem Zusammenhang die Frage zu verstehen ist. Eine die MP denn vorweisende Frage ist dadurch gekennzeichnet, dass der Sprecher etwas über den Sachverhalt zu wissen glaubt. Die Frage wird somit eine Kontrollfrage. Deswegen drücken Entscheidungsfragen mit denn immer Zweifel aus (Kriwonossow 1977: 62), und die MP weist den logischen Zusammenhang des Dialoges auf, der auf Deutsch immer ausgedrückt werden soll, eventuell mit einer MP (Becker 1976: 8). Die Kommunikationssituation ist dreigeteilt: Wenn der Sprecher des Satzes (9) nicht darüber nachdenkt, dass Fische im Behälter sein können, und anschließend von seinem Partner die Auskunft bekommt, dass es der Fall ist, kann er die Frage stellen. Also hat denn die Funktion, die Überraschung des Sprechers auszudrücken, und gleichzeitig kontrolliert er mit der Frage, dass er die Nachricht richtig verstanden hat. Wenn er ohne denn gefragt hätte, könnte er mit anderen Mitteln (z. B Intonation) zeigen, dass er überrascht war, aber ohne Hilfe von anderen Mitteln wäre die Frage überflüssig, da er ja eben die Auskunft bekommen hatte, dass es Fische im Behälter gibt.

Diesen Satztyp gibt es auch in der Variante „Rhetorische Frage“ (10). Denn fungiert hier genauso wie sonst. Doch enthält der Satz oft eine Anklage oder Kritik an den Gesprächspartner.

Bei diesem Satztyp gibt es faktisch nur eine Übersetzungsmöglichkeit, nämlich das dänische $d a$ : 
(9a) Er der da fisk derinde?

Wenn es sich um eine rhetorische Frage handelt, gibt es auch die im Dänischen verbreitete Möglichkeit, den Satz zu einem exklamatorischen Satz mit zwei Modalverben umzuschreiben:

(10a) Du må (da) være blevet tosset!

Noch eine die illokutive Funktion betonende Möglichkeit: Auf Dänisch gibt es die fast idiomatische Redewendung; sig mig (sag mir):

(10b) Sig mig, er du (da) blevet tosset?

Diese Umschreibung des Satzes gilt generell für alle deutsche die MP denn vorweisenden Fragen, und sie ist sehr typisch für die dänische Sprache.

Zusammenfassend gibt es auf Dänisch zwei unterschiedliche MP, während es auf Deutsch nur eine gibt. Die dänischen sind aber komplementär verteilt, so dass $d a$ überwiegend in unbetonten und rhetorischen Fragen und $s a ̊$ meist in betonten Fragen verwendet wird, ohne dass $s a ̊$ in unbetonten Fragen ausgeschlossen werden kann.

\subsection{Etwa}

(11) Soll man etwa dem armen Inspektor,..., das kleine Zubrot verweigern? (S.11)

(12) War es etwa auch dieser Wirklichkeitsblick, der aus Ihnen einen Befürworter des großen Lauschangriffs gemacht hat? (S. 3)

\subsubsection{Entscheidungsfragen}

Etwa ist eine MP, die dem Empfänger einen Hinweis gibt, wie er die Frage beantworten soll. Etwa kommt nur in Entscheidungsfragen vor (König 1977: 125). Der Kommunikationsprozess, in dem die MP vorkommt, ist dreigeteilt:

1. Der Sprecher bekommt eine Information, der er nur schwierig glauben kann, oder die er nicht ohne Weiteres annehmen kann. Im Beispiel (11) ist die Information, dass jemand die Regeln ändern will, so dass der Inspektor schlechter gestellt wird.

2. Die Frage wird gestellt.

3. Ein Nein wird erwartet, so dass der Empfänger zur Kenntnis gibt, dass er derselben Meinung wie der Sprecher ist. 


\section{4}

Dadurch dass die Frage überhaupt gestellt wird, macht der Sprecher klar, dass er befürchtet, dass der Empfänger anderer Meinung ist, dass er die Aussage des Sprechers bestätigen und sich dadurch tatsächlich in Widerspruch zum Sprecher stellen wird. Das könnte den Sprecher enttäuschen. Eine negative Antwort wird erwartet (Helbig 1977: 39). Der Gebrauch von etwa hängt nicht von der Wahrscheinlichkeit des Satzes ab, jedoch aber von den Wünschen und Bewertungen des Sprechers. Es besteht auch keine größere Wahrscheinlichkeit dafür, dass die Antwort tatsächlich verneinend wird, die Antwort kann ebenso gut positiv sein, aber der Empfänger muss auf jeden Fall seine Position erklären (Thurmair 1989: 172).

Die Kommunikationssituation muss allerdings nicht dreigeteilt sein: Die dritte Stufe könnte ausgelassen und die Frage ironisch gestellt werden, wodurch die Frage sich in eine rhetorische Frage verwandelt, worauf keine Antwort erwartet wird. Oder aber der Empfänger hat vorhin eine Frage gestellt, die der Sprecher als absurd einstuft (König 1977: 127). Das könnte im Beispiel (11) der Fall sein. Um seine Position zu verfestigen stellt der Sprecher dann die Frage mit etwa. Dieses Wort unterstreicht die empfundene Absurdität.

Im Beispiel (12) zeigt etwa auch welche Antwort erwartet wird. Je nach der Intonation könnte hier eine positive Antwort erwartet werden. In diesem Fall kann man die Frage ein wenig anders interpretieren: Der Satz drückt nur Zweifel und Unsicherheit aus. Der Inhalt des Satzes ist eine Möglichkeit, nicht nur eine verwerfliche Idee.

Die gleiche Funktion wird auf Dänisch durch die Kombination vel ikke wahrgenommen. Es ist bemerkenswert, dass man auf Dänisch eine Negation verwenden muss, um seinen Widerwillen gegen das Verhältnis auszudrücken, während man auf Deutsch geschickt die Gedanken des Empfängers „manipulieren“ kann, ohne explizit eine Negation zu verwenden. Natürlich kann man auch auf Deutsch eine Negation einsetzen, aber man muss es nicht. Auf Dänisch gibt es auch die Möglichkeit, die Negation zu vermeiden. Man könnte das Modalwort virkelig nutzen (wie im Beispiel (11)). Durch dieses Wort entsteht derselbe Effekt bei dem Empfänger, jedoch mit der Konnotation, dass die Befürchtungen des Sprechers eher Tatsache sind: Der Empfänger ist anderer Meinung als der Sprecher. Doch kann der Empfänger immer noch die Frage verneinen, 
aber dann ist die Wahrscheinlichkeit für einen nachfolgenden adversativen Satz größer. Der Empfänger muss seine Position erklären.

Eine Paraphrase ist auch in diesem Zusammenhang möglich: kan det virkelig vore rigtigt, at... Diese Paraphrase verleiht dem Zweifel des Sprechers und der negativen Haltung der Aussage gegenüber Ausdruck. Es wird ein deutliches Nein vom Empfänger antizipiert, sonst hat der Sprecher schon implizit gesagt, dass er gegen die Antwort Einwände vorbringen möchte.

Im Beispiel (12), wo das Wort auch zugleich auftaucht, könnte das Dänische auch die Konstruktion ikke også als Äquivalenz vorweisen. Dann wird eine positive Antwort erwartet.

Wenn man den Inhalt vom Beispiel (12) als eine nicht verwerfliche Möglichkeit ansieht, könnte hier auch das dänische Wort måske angemessen sein:

(12a) Var det måske også dette virkelighedssyn, der gjorde Dem til en repræsentant for det store lytteangreb?

Die Übersetzung ins Dänische hängt also vom Kontext und von der Interpretation des Satzes ab.

\subsection{Ja}

(13) Man hätte sich ja auch denken können, ... (S. 5)

(14) ...deshalb ja das archaische Bilderverbot. (S. 1)

(15) Wir wollen an dieser Stelle ja eigentlich keine alten Kamellen aufwärmen. (S. 1)

(16) Komm ja nicht zu spät.

(17) Es regnet ja!

(18) Du weinst ja!

\subsubsection{Deklarative Sätze}

In der Zeitung habe ich nur Beispiele mit $j a$ in deklarativen Sätzen gefunden. $\mathrm{Ja}$ in dieser Position zeigt dem Empfänger, dass die Aussage Wissen beschreibt, das beiden Gesprächspartnern bekannt sein dürfte. Der Sinn der Aussage ist es, dieses Verhältnis in die Erinnerung des Empfängers zu bringen, so dass eine gemeinsame Basis für den weiteren Verlauf der Kommunikation der beiden Gesprächspartner erstellt wird. 


\section{6}

Damit wird auf die Verbindung zwischen den beiden Gesprächspartnern hingewiesen, jedoch mit der Unterstellung, daß die Aussage nicht nur dem anderen bekannt sein sollte, sondern auch allen anderen Menschen. Die Aussage hat einen fast allgemeinbekannten Wert (Becker 1976: 9). Wenn man dagegen einen Satz zu einem gerade besprochenen Thema äußert, wäre die MP ja fehl am Platz, da es in diesem Fall offensichtlich ist, dass die Aussage beiden Gesprächspartnern bekannt ist. Gleichzeitig kann eine Aussage dem Empfänger so offenkundig sein, dass der Sprecher diese Aussage mit einem die MP ja beinhaltenden Satz fast schroff zurückweist (Bublitz 1975: 144-5). Dem könnte der Fall (13) ein Beispiel sein. In diesem Satz wird ein Signal zum Empfänger gegeben, dass es erstens Basiswissen sein dürfte, und dass er deswegen zweitens dem Sprecher beistimmen sollte. Im Beispiel (14) könnte der Sprecher die Intention hegen, den Sprecher an die Verhältnisse der Sache und an die dahinter liegenden Gründe zu erinnern, indem vorausgesetzt wird, dass der Empfänger die Informationen innehat, an die der Sprecher mit seiner Äußerung anspielt.

Die MP ja kann auch von einem anderen kommunikativ-pragmatischen Winkel betrachtet werden, wo die MP die Funktion hat, die Äußerung in der Argumentation festzulegen, und dadurch die Aussage in Relation zu den geltenden Umständen zu setzen (Brausse 1986: 211). Damit könnte Beispiel (14) ein Argument in der Diskussion sein, warum man überhaupt ein Bilderverbot eingeführt hat, aber nur dann wenn der Satz in Verbindung mit einer These geäußert wird. Ja legt den Satz in einem argumentativen Zusammenhang fest. Der Umstand, dass die Aussage bekannt ist, ist nicht dafür entscheidend, dass man den Satz als Argument verwenden kann, aber da die Aussage durch den Gebrauch von der MP $j a$ allgemeines Wissen sein sollte, kann der Satz als Argument dafür verwendet werden, dass die erwähnte These glaubwürdig ist (Brausse 1986: 214).

Diese MP kann auch eine manipulatorische Funktion haben in dem Fall, dass der Empfänger nicht das Wissen innehat, das der Sprecher erwartet (Lütten 1979: 38). Der Sprecher kann dadurch das Wissen des Empfängers in Frage stellen, dass er eine Aussage mit $j a$ hervorbringt, obwohl er vielleicht schon weiß, dass der Sprecher überhaupt keine Ahnung zum Thema hat. Dadurch wird der Satz zu einer Art Arroganz von Seiten des Sprechers - der will sein Wissen als allgemein bekannt darbringen und dadurch vielleicht den Empfänger in Verlegenheit 
bringen, weil er das „Allgemeinwissen“ nicht kennt. In dieser Situation wird der Empfänger gezwungen, seine Wissenslücke durch eine Gegenfrage wie „Welches Bilderverbot?“ (zu (14)) bloßzustellen, oder er muss so tun, als ob er schon einverstanden ist. Natürlich besteht auch die Möglichkeit, dass der Sprecher wirklich denkt, dass der Empfänger Bescheid weiß, und verwendet deswegen die MP. Also kann $j a$ in deklarativen Sätzen in einer sozialen und positiven Weise gebraucht werden, indem der Sprecher eine gemeinsame Wissensbasis anknüpft, oder in einer manipulatorischen Weise - mit oder ohne Absicht.

Auf Dänisch ist die Äquivalenz jo. Diese beiden MP ja und jo entsprechen einander völlig in dieser Hinsicht. Sonst könnte man auch eine Paraphrase wie som $d u$ ved da einfügen, wo eine Auskunft gegeben wird, mit der der Empfänger schon einverstanden ist:

(14a) ...derfor (som du ved) også det arkaiske billedforbud (, som du ved).

Diese Paraphrase könnte auch in Sätzen wie (15) verwendet werden, aber in den meisten Fällen würde man jo verwenden. Die Paraphrase ist in Sätzen wie (13) nicht genauso idiomatisch, wo der Inhalt eher von philosophischem Charakter ist, wo der Sprecher eher auf die Vernunft hinweist als auf ein tatsächliches Faktum. Generell ist jo die gültige Entsprechung.

\subsubsection{Imperativische Sätze}

Da die MP $j a$ in interrogativen Sätzen so gut wie gar nicht vorhanden ist, werden diese Satztypen hier übersprungen. In imperativischen Sätzen hat die MP die Funktion den illokutiven Modus „Aufforderung“ zu unterstreichen, und dadurch auch den Befehl/ die Aufforderung. Wenn $j a$ in dieser Art und Weise vorkommt, modifiziert es nicht bloß die Illokution, verändert sie aber vielmehr in eine Drohung oder eine Warnung (Burkhart 1982: 356). In dieser Funktion ist die MP betont, was sonst nicht so oft vorkommt (16). Sie hat fast dieselbe Funktion wie der ethische Dativ:

(16a) Komm mir nicht zu spät!

Sie macht die Aussage persönlich: Wenn der Empfänger dem „Befehl“ nicht nachkommt, ist es fast eine persönliche Kränkung des Sprechers. Doch ist die Aussage mit ja ein bisschen objektiver als die Aussage mit dem ethischen Dativ. 


\section{8}

Auf Dänisch hat man in diesem Fall nicht die Möglichkeit, jo zu verwenden, dafür aber eine andere MP: $n u$. In der Aufforderung kann der paraphrasierte Inhalt von $j a$ nur vage erkannt werden: Es wird als Forderung angegeben, dass der Empfänger nicht zu spät kommen darf, aber gleichzeitig könnte man vielleicht annehmen, dass der Sprecher versucht, den Empfänger zum Beistimmen zu bewegen? In dieser logischinhaltsbezogenen Weise betrachtet, kann es wundern, dass man die Konsensuspartikel jo nicht einsetzen kann. Aber diese Unterstreichung der Aufforderung in Kombination mit einem Versuch, Einigkeit zu erzielen, haben wir auf Dänisch nicht. $N u$ macht den „Befehl“"zwar weniger schroff und eher eindringlich, aber es wird kein Versuch unternommen, Konsensus zu erzielen. Die Aufforderung könnte je nach der Intonation in ein Gebet verwandelt werden. Darüber hinaus haben wir eine oft gebrauchte Konstruktion:

(16b) Lad nu være med at komme for sent!

Die MP $n u$ tritt zwar immer noch auf, aber das Interessante an dieser Konstruktion ist, dass man ein Hilfsverb verwendet. $N u$ könnte ausgelassen werden. Der Satz mit lad hat denselben eindringlichen Inhalt wie der deutsche Satz mit ja.

Noch eine Möglichkeit, dieses Verhältnis auszudrücken, wäre endelig:

(16c) Kom (nu) endelig ikke for sent!

\subsubsection{Exklamatorische Sätze}

In exklamatorischen Sätzen hat $j a$ wieder eine andere Funktion: Sie zeigt die Überraschung des Sprechers über ein Verhältnis, das er nicht erwartet hatte. $J a$ in einem exklamatorischen Satz drückt aus, dass ein Verhältnis möglich aber nicht erwartet ist (Lindner 1991: 181). Diese Funktion kann auch von doch wahrgenommen werden, aber dann eher mit den Konnotationen, dass eine frühere Auffassung oder Äußerung widerlegt wird, wohingegen $j a$ vielmehr der unmittelbare Ausdruck dafür ist, dass das Geschehene unerwartet war. $J a$ ist Ausdruck für Überraschung, weil der Sprecher plötzlich erkennt, dass ein Verhältnis so ist, wie es ist (Burkhardt 1982: 353). Das ist am Beispiel (17) zu erkennen. Ja ist nicht der entscheidende Faktor dafür, dass der Satz als exklamatorisch aufzufassen ist. Die Intonation sorgt dafür. Aber wenn ja in den exklamatorischen Sätzen auftaucht, können sie als das Gespräch eröffnende Sätze 
aufgefasst werden; dies im Gegensatz zu deklarativen Sätzen, in denen $j a$ vorkommt. In den exklamatorischen Sätzen mit ja liegt nämlich manchmal eine versteckte Aufforderung zum Empfänger, das Verhältnis, über das der Sprecher überrascht ist, zu begründen (Brausse 1986: 217). In einem Satz wie (18) wird der Empfänger fast zu einer Erklärung, warum er weint, aufgefordert.

Kommunikativ kann man versuchen, die exklamatorische Aussage in einen Konditionalsatz einzubinden (Brausse 1986: 216). Somit kann (17) in eine fiktive Situation eingefügt werden: Der Empfänger schlägt einen Spaziergang vor. Der Sprecher ist der Meinung, dass man beim Regen nicht spazieren geht. Weil es regnet, wird der Vorschlag zurückgewiesen. Der Konditionalsatz könnte so lauten: Wenn es regnet, gehen wir nicht spazieren. Der exklamatorische Satz wird dadurch zu einem Argument dafür, dass man nicht spazieren gehen sollte. Impliziert liegen der ganze Konditionalsatz und die Schlussfolgerung in der Aussage (17); man sollte nicht spazieren gehen. Dieselbe Funktion könnte man mit der MP doch erreichen.

Auf Dänisch gibt es die Entsprechung jo. Eine andere Möglichkeit wäre minsandten oder

(17a) Jamen dog, det regner.

Die beiden letzen Möglichkeiten sind nicht akzeptabel in dem Fall, wo es um einen versteckten Konditionalsatz geht: Sie sind nur als Ausdruck für die Überraschung verwendbar.

\subsection{Schon}

(19) Die Nacht, sagen sie im Haus, kann schon mal zur Qual werden. (S. 12 Leben)

(20) Irgendwie werden wir uns schon durchwursteln. (S. 15 Leben)

(21) Ein bisschen komplizierter liegt der Fall schon (S. 52)

(22) Wer weiß schon noch, dass...? (S. 1)

(23) Wer will schon gern begreifen, dass ein Bundeshaushalt ähnlich wie der eigene funktioniert. (S. 15 Leben)

(24) Was man für hohe Herren sieht, wenn man schon mal spazieren geht! (S. 20) 


\subsubsection{Deklarative Sätze}

Erstens kann schon in deklarativen Sätzen mit Hinweis auf die Zukunft vorkommen, wie Beispiel (19) und (20). In diesen Fällen drückt schon aus, dass die Äußerung richtig ist, und dass der Sprecher so autoritär ist, dass man ihm glauben muss. Der Sprecher ist sich ganz sicher, dass der Inhalt des Satzes eintreten wird (Borst 1985: 58). Schon soll Zuversicht zum Sprecher erwecken, was sich ferner darin zeigt, dass schon auch manchmal als Trost oder Hoffnung funktionieren soll.

In diesem Fall benutzt man die MP nok, oder in Fällen wie (19) vielleicht godt. Diese MP könnte auch in (20) gebraucht werden, unter der Voraussetzung dass man eine Umschreibung des Satzes mit kan statt skal macht, welches Wort auch in (19) gebraucht wird. Skal kann nicht mit godt kombiniert werden. In (19) wird nok in einer Unheil verkündenden Situation verwendet: Der Empfänger wird beinahe gewarnt. Aber auch hier bewirkt nok, dass der Sprecher so viel Vertrauen erweckt, dass man ihm glauben muss. Man soll die Warnung ernst nehmen.

Togeby (1979: 19-20) schreibt über nok, das diese MP den Schwerpunkt des Satzes verschieben kann. Damit wird gemeint, dass die MP eine wichtige Information über den Sachverhalt hinweg verschieben kann. Nok weist über den Satz hinweg und zeigt, wie der Empfänger sich zum Gesagten verhalten soll, und zwar mit Vertrauen. Togeby teilt auch die Bedeutungen von nok in feststellende, bewertende und regulierende Bedeutung auf. Nok hat hier eine feststellende Funktion, da man Togeby zufolge die Bedeutung so paraphrasieren kann: So wahrscheinlich, dass man glauben kann.

Außer diesen Übersetzungsmöglichkeiten gibt es auch die Möglichkeiten faktisk (nok), und da bzw. da i hvert fald (Baunebjerg/ Wesemann 1983: 124). Letztgenannter Vorschlag ist wahrscheinlich noch ,aufmunternder" und zeigt, dass der Sprecher seiner Sache noch sicherer als mit der Verwendung nok ist.

Zweitens: Wenn schon in einem deklarativen Satz ohne Bezug auf die Zukunft wie in (21) steht, drückt die MP eine Bestätigung des Vorherigen aus. Schon zeigt, dass der Sprecher weitgehend mit dem Gesagten einverstanden ist, jedoch gibt es Argumente, die das Gesagte einschränken können. Die Richtigkeit der Aussage wird generell bestätigt, wird aber im Einzelfall verneint (Bublitz 1978: 92). Deswegen wird der nächste Satz oft mit einer adversativen Konjunktion angefangen, die die Aussage 
einschränkt. Diese Einwendungen spielen jedoch keine große Rolle für die Konklusion des Dialoges: Trotz der Einwände kann sich der Gesprächspartner einverstanden erklären.

Diese Funktion wird auf Dänisch durch die MP godt nok, rigtignok eller ganske vist wahrgenommen. Die MP godt nok bestätigt eher den vom Gesprächspartner geäußerten Satz als die anderen Möglichkeiten, und der nachfolgende adversative Satz wiegt nicht so schwer in der gesammelten Konklusion des Gespräches, während ein Satz mit einer der anderen Möglichkeiten eher Gegenargumente hervorruft:

(21a) Det er godt nok et svært tilfælde/ Godt nok er det et svært tilfælde, men...

(21b) Det er ganske vist et svært tilfælde, men...

\subsubsection{Entscheidungsfragen}

In Entscheidungsfragen wie (22) und (23) ist schon ein illokutiver Faktor, der bewirkt, dass die Frage in eine rhetorische Frage gewandelt wird, auf die eine negative Antwort erwartet wird (Thurmair 1989: 201). Die Frage an sich ist negativ oder pessimistisch gedreht, und in ihrer Grundstruktur ist es eine rhetorische Frage, da man keine Antwort erwartet oder aber die einleuchtende Antwort „keiner“ (Meibauer 1994: 192). In (23) ist der Sprecher einer bestimmten Meinung über z. B. Haushalt, und durch seine Frage argumentiert er im Grunde für seine Meinung: Sie ist eine Begründung dafür, dass Leute die Sachen nicht so erkennen wollen, wie sie sind. Wenn der Empfänger auf diese Frage eine (andere) Antwort gibt, ist es wider Erwarten und wider die Regeln der Kommunikation. Eine Frage mit schon ruft eine negative Antwort hervor, und dadurch eine Erklärung des Einverständnisses auf Seiten des Empfängers - wie bei etwa.

Dieser Funktion entsprechen auf Dänisch også oder $d a$ (også).

(22a) Hvem husker da også stadig på at...?

Eine andere Möglichkeit wäre eine Paraphrase mit einem Modalverb wie ville oder skulle.

(22b) Hvem skulle (da også) huske på...

Oder:

(23a) Hvem skulle kunne forstå, at landets økonomi fungerer som ens egen. 
152

Eine andere Methode, das Unwahrscheinliche der Aussagen zu unterstreichen und damit eine negative Antwort hervorzurufen, wäre wiederum die durative Form:

(22c) Hvem går rundt og husker...

\subsubsection{Konditionalsätze}

Schon kann ein Element in der Kondition eines Konditionalsatzes sein. Wie immer in Konditionalsätzen wird der Inhalt des Wenn-Satzes vorausgesetzt, ist aber keine gegebene Sache, während die Sachverhalte im Hauptsatz für gegeben angesehen werden, wenn der Wenn-Satz erfüllt wird. Schon verstärkt diese Funktionen wie in (24). Wolski (1983: 466) nennt dieses schon für ,DAS schon der A-FORTIORI-ARGUMENTATION“.

Die einzige Möglichkeit, dies auf Dänisch auszudrücken ist alligevel. Mit diesem Wort wird die etwas distanzierte Haltung des Sprechers dem Verhältnis gegenüber ausgedrückt: Es ist fast ungelegen die Handlung auszuführen, aber wenn man sich schon die Mühe gibt, kann man ebenso gut das Beste davon bekommen. Es ist schwer die Konnotationen von schon in dieser Funktion zu erfassen, aber die Konnotationen werden wohl am besten durch alligevel weitergegeben.

\section{Konklusion}

Da die MP bis vor Kurzem ein so unerforschtes Gebiet in der Sprachwissenschaft ausmachten, gibt es immer noch nicht so viel Literatur zu dem Thema, doch gibt es genug dazu, dass man sich einen Überblick über ihre Funktionen und Bedeutungen für die Kommunikation bilden kann. Und sie sind sehr wichtig für die Kommunikation, warum man sich im z. B. Fremdsprachenunterricht intensiver damit auseinander setzen sollte. Es ist auch ein schwieriges Gebiet, da die MP kommunikativ wichtig sind, jedoch sprachlich schwer zu erfassen und zu erklären sind. Ein Deutsch lernender Däne könnte deswegen Nutzen von einem MPWörterbuch haben, wo er nachschlagen kann, welche Funktionen diese kleine Wörter haben. Dadurch kann er vielleicht einfacher ihre Verwendungsmöglichkeiten lernen, und im Anschluss daran die Wörter selber brauchen. 
Bis jetzt gibt es aber kein solches Nachschlagewerk über die dänischen und deutschen MP, zumal die dänischen MP nicht so ausführlich behandelt worden sind. Ausgehend von den Prinzipien der oben stehenden Analyse wäre es möglich alle MP der beiden Sprachen zu analysieren und die Ergebnisse festzuhalten. Damit hätte man fast das DeutschDänisch Wörterbuch über die MP. Wenn man diese MP alle analysiert hat und ihre Entsprechungen in den beiden Sprachen gefunden hat, könnte man vielleicht auch überlegen, ob sie in einem ganz normalen DeutschDänisch Wörterbuch in Bezug auf ihre Funktionen ausführlicher behandelt werden sollen.

\section{Literaturliste}

Abraham, Werner (1986). Die Bedeutungsgenese von Modalpartikeln. In Groninger Arbeiten zur germanistischen Linguistik 27. 1-44.

Andersen, John Edelsgaard (1989). Sproghandlinger - på dansk. København: Dansklærerforeningen.

Baunebjerg, Gitte/Wesemann, Monika (1983). Partikelwörterbuch Deutsch-Dänisch, Dänisch-Deutsch. Ein Arbeitsbericht. In Weydt, Harald (1983): Partikeln und Interaktion. Tübingen: Max Niemeyer Verlag. 119-129.

Becker, Norbert (1976). Die Verknüpfungspartikeln ,denn, mal, doch` und andere. In Zielsprache Deutsch 7. 6-12.

Borst, Dieter (1985). Die Affirmativen Modalpartikeln doch, ja und schon. Ihre Bedeutung, Funktion, Stellung und ihr Vorkommen. Tübingen: Niemeyer.

Brausse, Ursula (1986). Zum Problem der sogenannten Polyfunktionalität von Modalpartikeln. Ja und eben als Argumentationssignale. In Zeitschrift für Phonetik, Sprachwissenschaft und Kommunikationsforschung 39. 206-223.

Bublitz, Wolfram/Roncador, Manfred von (1975). Über die deutsche Partikel ja. In Studien zur deutschen Grammatik 2. Syntaktische und semantische Studien zur Koordination. Tübingen: Verlag Gunter Narr. 139-190.

Bublitz, Wolfgang (1978). Ausdrucksweisen der Sprechereinstellung im Deutschen und Englischen. Tübingen: Max Niemeyer Verlag.

Burkhardt, Armin (1982). Die kommunikativen Funktionen von ,,ja“ und ihre lexikographische Beschreibung in Wörterbüchern. In Muttersprache 92. 337-361.

Dittmann, Jürgen (1980). AUCH und DENN als Abtönungspartikeln. In ZGL. 51-73.

Helbig, Gerhard (1977). Partikeln als illokutive Indikatoren im Dialog. In Deutsch als Fremdsprache 14. 30-44.

Heinrichs, Werner (1981). Die Modalpartikeln im Deutschen und Schwedischen. Tübingen: Max Niemeyer Verlag. 


\section{4}

Helbig, Gerhard (1994). Lexikon deutscher Partikeln. Berlin: Langenscheidt, Verlag Enzyklopädie 3. Auflage.

Kirstein, B.( 1983). Partikeln und Sprechsituation. In Weydt, Harald (1983). Partikeln und Interaktion. Tübingen: Max Niemeyer Verlag. 213-225.

König, Ekkehard (1977). Modalpartikeln in Fragesätzen. In Weydt, Harald (1977). Aspekte der Modalpartikeln. Studien zur deutschen Abtönung. Tübingen: Max Niemeyer Verlag. 115-130.

Kriwonossow, Alexej (1977). Die modalen Partikeln in der Gegenwartssprache. Verlag Alfred Kümmerle.

Krivonosov, A. (1983). Zur Rolle der Partikeln bei der ,Einspannung des Sprachmaterials‘. In Weydt, Harald (1983). Partikeln und Interaktion. Tübingen: Max Niemeyer Verlag. 40-45.

Lindner, Katrin (1991). „Wir sind ja doch alte Bekannte“. The use of German ja and doch as modal particles. In Abraham, Werner (Hrsg.): Discourse Particles. Amsterdam/ Philadelphia: John Benjamins Publishing Company. 163-201.

Lütten, Jutta (1979). Die Rolle der Partikeln doch, eben und ja als Konsensus-Konstitutiva in gesprochener Sprache. In Weydt, Harald (Hrsg.): Die Partikeln der deutschen Sprache. Berlin, New York: Walter de Gruyter. 30-38

Meibauer, Jörg (1994). Modaler Kontrast und konzeptuelle Verschiebung. Studien zur Syntax und Semantik deutscher Modalpartikeln. Tübingen: Max Niemeyer Verlag.

Thurmair, Maria (1989). Modalpartikeln und ihre Kombinationen. Tübingen: Max Niemeyer Verlag.

Togeby, Ole (1979). Nok. In Mål og Maele 3. 18-23.

Weydt, Harald (1969). Abtönungspartikeln. Die deutschen Modalwörter und ihre französischen Entsprechungen. Bad Homburg v.d.H.: Verlag Gehlen.

Wolski, Werner (1983). Die Modalpartikel schon in Wörterbüchern und linguistischen Untersuchungen. Ein Beitrag zur praktischen Lexikologie. In Germanische Linguistik 14. 453-486.

\section{Zeitungen}

Die Zeit fra den 28.10.99

Politiken fra den 15. og 16.11.99 